\title{
VALORES DE REFERÊNCIA LOCAL E AVALIAÇÃO DA CONTAMINAÇÃO POR ZINCO EM SOLOS ADJACENTES A ÁREAS MINERADAS NO MUNICÍPIO DE VAZANTE-MG ${ }^{(1)}$
}

\author{
Meubles Borges Júnior ${ }^{(2)}$, Jaime Wilson Vargas de Mello ${ }^{(3)}$, Carlos Ernesto G. R. \\ Schaefer $^{(3)}$, Tânia Mara Dussin ${ }^{(4)}$ \& Walter Antônio Pereira Abrahão(5)
}

\begin{abstract}
RESUMO
A contaminação dos solos por metais pesados é um problema atual que requer abordagem adequada, tendo em vista as necessidades da sociedade moderna. Em áreas de mineração, a avaliação da contaminação de solos constitui ferramenta importante para o gerenciamento de áreas degradadas. Este trabalho foi desenvolvido com o objetivo de estabelecer valores de referência local (VRL) para Zn em solos adjacentes a áreas mineradas no município de Vazante-MG, tendo em vista avaliar a contaminação ambiental. Foram coletadas amostras de perfis de solos remanescentes em área mineralizada, a jusante e montante dessa, nas profundidades de 0-2, 2-5, 5-10, 10-20, 20-50, 50-100 e 100-150 cm. Foram determinados os teores totais de $\mathrm{Zn}$, bem como as formas disponíveis (Mehlich-3 e DTPA). Optou-se por determinar o VRL a partir da média ponderada dos teores de $\mathrm{Zn}$ nas camadas de 0 - $20 \mathrm{~cm}$ dos perfis a montante. De posse desse valor, calcularam-se o fator de contaminação (FC) e o índice de geoacumulação (IGeo) para cada perfil. O VRL obtido para Zn foi de $1.262 \mathrm{mg} \mathrm{kg}^{-1}$, que é muito alto em relação aos padrões de qualidade, sugerindo monitoramento e restrição ao uso agrícola e residencial dos solos na área. Não obstante, esse valor é inferior aos valores de intervenção (VI's) para as áreas industriais adotados no Estado de São Paulo, EUA e Canadá. Os solos das áreas mineralizadas apresentaram maiores valores para o fator de contaminação, com destaque para o perfil 5 localizado na área mineralizada em exploração (área minerada). De acordo com o IGeo, os perfis de solo a jusante foram classificados como não poluídos a moderadamente poluídos.
\end{abstract}

Termos de indexação: solos contaminados; valores orientadores, background de Zn.

\footnotetext{
(1) Trabalho apresentado no XXXI Congresso Brasileiro de Ciência do Solo, 2007 (Gramado, RS).

(2) Professor do Centro Universitário de Caratinga. Av. Moacyr de Mattos, 49. 35300-047 Caratinga, MG. E-mail: meubles@funec.br.

(3) Professor Associado do Departamento de Solos, Universidade Federal de Viçosa (UFV). Av. P.H. Rolfs, s/n. 36570-000 Viçosa, MG. Pesquisador CNPq. E-mail: jwvmello@ufv.br e carlos.schaefer@ufv.br

(4) Professor Associado do Departamento de Geologia, IGC. Universidade Federal de Minas Gerais (UFMG). Av. Antônio Carlos. 6627, Pampulha - Belo Horizonte, MG. Email: dussin@netuno.lcc.ufmg.br

(5) Professor Adjunto de Departamento de Solos, UFV. Av. P.H. Rolfs, s/n. 36570-000 Viçosa, MG. Email:wabrahao@ufv.br.
} 


\title{
SUMMARY: ESTABLISHINGLOCAL REFERENCE VALUESANDDETERMINING CONTAMINATION OF ZINC IN SOILS OF VAZANTE-MG
}

\begin{abstract}
Soil contamination by heavy metals is a current concern that requires a suitable approach, taking the needs of modern societies into account. In mined areas the evaluation of soil contamination is an important tool for the management of degraded areas. This study was carried out in order to establish local reference values ( $L R V)$ for $Z n$ in soils surrounding mining areas in the county of Vazante-MG (Brazil), with a view to evaluate the environmental contamination. Soil profiles were collected in the mining areas, and both upslope and downslope and sampled at depths of 0-2, 2-5, 5-10, 10-20, 20-50, 50-100 and 100-150 cm. Total concentrations and available forms (Melich-3 and DTPA) of Zn were determined. LRV was obtained by the mean of total $\mathrm{Zn}$ contents in the $0-20 \mathrm{~cm}$ layer of the profiles in upslope position. Based on the $L R V$, the contamination factor $(C F)$ and the geoaccumulation index (GI) were calculated for each profile. The LRV determined for Zn was $1262 \mathrm{mg} \mathrm{kg}^{-1}$, which is a very high content when compared to quality standard values and suggests monitoring and restrictions in the agricultural and residential soil use. Nevertheless, the LRV is lower than the intervention values for industrial areas established in the State of São Paulo, USA and Canada. The CF was higher in soils of the mined area, mainly in profile 5 of the mining area. Based on the GI, the profiles located downslope were classified as moderately contaminated to not contaminated.
\end{abstract}

Index terms: contaminated soils; guide values; Zn background level.

\section{INTRODUÇÃO}

A contaminação dos solos por metais pesados é preocupação mundial, tendo em vista as necessidades tecnológicas da sociedade moderna. As áreas de mineração, em particular, são drasticamente alteradas e, nesse contexto, a avaliação da contaminação dos solos é o primeiro passo para o adequado gerenciamento das áreas degradadas. A avaliação preliminar de uma área suspeita de contaminação é realizada com base em informações disponíveis (CETESB, 1999). A área é considerada contaminada se as concentrações de elementos ou substâncias de interesse estiverem acima de dado limite, denominado valor de intervenção, o qual indica risco potencial de efeito deletério sobre a saúde humana. Nesse caso, há necessidade de intervenção imediata na área, incluindo investigação detalhada e adoção de medidas emergenciais, tais como: restrição do acesso de pessoas à área e suspensão do consumo de água subterrânea (CETESB, 2001).

Os métodos utilizados pelas agências ambientais, para tomada de decisão sobre áreas suspeitas de contaminação, podem ser agrupados em: (i) aqueles que utilizam valores orientadores preestabelecidos, com ou sem diferenciação do uso do solo e (ii) aqueles que se baseiam na avaliação de risco caso a caso (Visser, 1994).

Em se tratando de metais pesados em solos, os valores orientadores de referência são definidos a partir do "background" da área. Para tanto, são considerados resultados de análises químicas dos teores totais (ou quase totais) em áreas pretensamente protegidas de efeitos antrópicos. De modo geral, os valores que indicam a necessidade de intervenção ou investigação das áreas suspeitas de contaminação também consideram os teores totais dos metais pesados nos solos (USEPA, 1996; VROM, 1994; CETESB, 2001).

Alguns autores questionam a validade de usar teores totais para fins regulatórios, tendo em vista que, na maioria dos casos de contaminação e avaliação de risco, o que realmente interessa é a fração biodisponível (Ribeiro Filho et al., 1999). No entanto, não há um método universal para avaliar a biodisponibilidade dos elementos-traço, de modo que a fração disponível pode ser muito diferente quando se considera a absorção por planta ou a ingestão de solo por criança (Marques et al., 2002). Diante disso, os autores consideram que os teores totais ou quase totais continuarão sendo considerados para fins regulatórios.

O principal problema de considerar teores totais é que esses não refletem a mobilidade ou a biodisponibilidade. Sabe-se que uma fração, geralmente pequena, do teor total de um metal pesado no solo pode ser mobilizada por processos de lixiviação ou absorvida pelas plantas. Com relação ao Zn, de 1 a 2 $\%$ do teor total pode ser considerado biodisponível em solos do Cerrado (Marques et al., 2002). Portanto, na fase de investigação de valores anômalos em áreas suspeitas deve-se atentar também para análises de teores disponíveis no solo, além dos teores totais ou quase totais.

Diante das dificuldades de realizar bioensaios; ou seja, utilizar seres vivos para determinar os teores biodisponíveis de metais pesados em solos, vários métodos de análise química vêm sendo estudados em 
diferentes ambientes e condições pedológicas. Dentre os vários extratores utilizados para avaliar a disponibilidade para plantas de micronutrientes e metais pesados em solos, destacam-se os métodos que utilizam complexantes, como o DTPA e o extrator de Mehlich-3. De modo geral, os teores extraídos por DTPA têm revelado boas correlações com as quantidades absorvidas pelas plantas e o método foi padronizado para extração dos teores disponíveis de $\mathrm{Cu}, \mathrm{Zn}$ e Fe na Itália (Rauret, 1998). Os extratores Mehlich-1 e, mais recentemente, o Mehlich-3 (Mehlich, 1984) vêm sendo utilizados no Brasil com relativo sucesso.

A investigação em áreas anômalas, suspeitas de contaminação, atende a vários objetivos, dentre eles avaliar a extensão da contaminação e buscar pistas sobre a origem da contaminação, se natural ou antrópica. Nesse sentido, o presente trabalho é um estudo de caso que visa a contribuir com nova abordagem para investigações de contaminação e avaliação da contribuição antrópica em áreas contaminadas. Em outro trabalho (Borges Júnior et al., 2008), realizado na área, esses aspectos foram avaliados a partir de análises químicas seqüenciais e distribuição dos teores de $\mathrm{Zn}$ em perfis de solo. A presente abordagem propõe a derivação de "valores de referência local", a partir de teores totais e disponíveis de Zn, e o uso de alguns índices geoquímicos na investigação da área.

O presente trabalho objetivou estabeler "valores de referência local" para Zn nos solos em áreas próximas à mineração, na região de Vazante-MG e avaliar a contaminação local por comparação dos valores obtidos com os encontrados na literatura e também por meio do fator de contaminação e índice de geoacumulação.

\section{MATERIAL E MÉTODOS}

\section{Descrição da Área}

A região de Vazante - MG apresenta áreas anômalas, com altos teores de $\mathrm{Zn}$ e $\mathrm{Pb}$, destacando-se como maior distrito produtor de $\mathrm{Zn}$ do Brasil. O município localiza-se na porção N-NW do Estado de Minas Gerais, entre as cidades de Paracatu e Patos de Minas (Figura 1). Os minérios compõem-se, principalmente, de willemita ${ }^{(1)}$ e calamina ${ }^{(2)}$. A mineralização ${ }^{(3)}$ está associada a rochas carbonáticas da Formação Vazante, com corpos de minério formados por veios associados a falha longitudinal de direção NE. Trata-se de formação restrita à seqüência predominantemente dolomítica (Rigobello et al., 1988). O minério é retirado de duas lavras específicas: i) a mina a céu aberto responsável pela produção de calamina e ii) a lavra subterrânea responsável pela produção de willemita.

\section{Coleta e caracterização das amostras de solos}

As amostras foram coletadas em solos remanescentes na área mineralizada e a jusante e montante nas áreas sob influência da mineração. Os locais de coleta foram selecionados conforme descrito por Borges Júnior et al. (2008). Em cada perfil de solo, foram retiradas amostras a sete profundidades $(0-2$; $2-5 ; 5-10 ; 10-20 ; 20-50 ; 50-100$ e $100-150 \mathrm{~cm})$, com exceção do perfil 4, em que não foi coletada amostra na profundidade de $100-150 \mathrm{~cm}$. As amostras foram secas ao ar, destorroadas e tamisadas em peneiras de 2,0 $\mathrm{mm}$ de abertura de malha, procedendose às análises de caracterização física e química, conforme Embrapa (1999), e determinações dos teores totais e disponíveis de Zn.

\section{Determinação dos teores de $\mathrm{Zn}$}

Para determinar os teores totais, utilizaram-se 0,5 $\mathrm{g}$ de solo triturado em almofariz de ágata e mistura de $\mathrm{HNO}_{3}(8 \mathrm{~mL}), \mathrm{HCl}(5 \mathrm{~mL})$ e $\mathrm{HF}(5 \mathrm{~mL})$ com digestão em microondas modelo ETHOS PLUS, Labstation - Milestone. A digestão foi programada para atingir $160{ }^{\circ} \mathrm{C}$ em $6 \mathrm{~min}$, mais 6 min para atingir $210{ }^{\circ} \mathrm{C}$, permanecendo nesta temperatura por $20 \mathrm{~min}$. Após a digestão, adicionaram-se $5 \mathrm{~mL}$ de $\mathrm{H}_{3} \mathrm{BO}_{3}$ a $1 \%$ completando o volume para $100 \mathrm{~mL}$. Além da determinação da concentração total, foram realizadas extrações com DTPA (Lindsay \& Norvel, 1978) e Mehlich-3 (Mehlich, 1984), para a obtenção das formas disponíveis. A concentração de Zn nos extratos foi determinada por espectrofometria de absorção atômica.

Foram calculadas as médias ponderadas dos teores de $\mathrm{Zn}$ (totais e disponíveis) para as profundidades de 0 - 20 cm e 20 - $150 \mathrm{~cm}$, em cada perfil. Com as médias ponderadas dessas camadas, foi calculado o fator de acumulação, que é dado por:

$$
\mathrm{FA}=\mathrm{C}_{\mathrm{Zn}(0-20 \mathrm{~cm})} / \mathrm{C}_{\mathrm{Zn}(20-150 \mathrm{~cm})}
$$

em que $\mathrm{C}_{\mathrm{Zn}(0 \text { a } 20 \mathrm{~cm})}$ é a concentração do elemento na camada de 0 - $20 \mathrm{~cm}$ e $\mathrm{C}_{\mathrm{Zn}(20 \text { a } 150 \mathrm{~cm})}$ é a concentração do mesmo na camada de $20-150 \mathrm{~cm}$.

O FA indica quantas vezes a camada superficial está mais concentrada do que a camada subsuperficial, no que se refere aos teores de $\mathrm{Zn}$.

\footnotetext{
(1) Silicato de zinco cuja fórmula química é $\mathrm{Zn}_{4}\left(\mathrm{Si}_{2} \mathrm{O}_{7}\right)$.

(2) Silicato de zinco, também chamado de hemimorfita, cuja fómula química é $\mathrm{Zn}_{4}\left(\mathrm{Si}_{2} \mathrm{O}_{7}\right)(\mathrm{OH}) \cdot 2 \mathrm{H}_{2} \mathrm{O}$.

(3) No presente trabalho, será considerado o termo “mineralização" para se referir a depósitos de minérios e "mineração" para indicar a exploração destes minérios.
} 


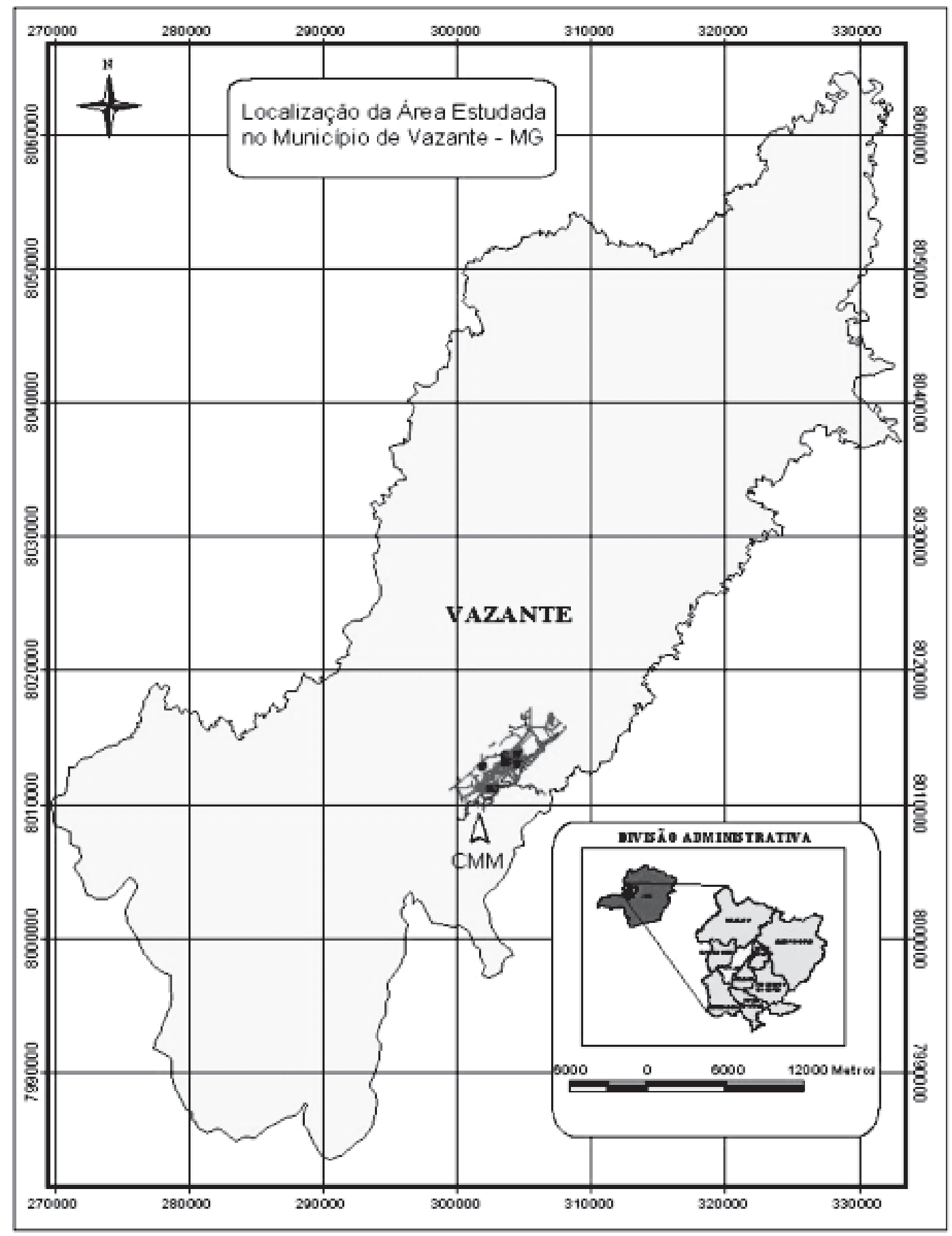

Figura 1. Mapa de localização do município de Vazante, com destaque para a área de mineração de zinco.

\section{Obtenção dos valores de referência}

Para obter os valores de referência local (VRL), foram consideradas as seguintes possibilidades: i) utilização dos teores totais ou disponíveis, ou ainda, da fórmula do método holandês (CETESB, 2001) que considera os teores de argila e matéria orgânica; ii) adoção das profundidades de $0-20 \mathrm{~cm}, 0-150 \mathrm{~cm}$ ou
20 - $150 \mathrm{~cm}$; iii) utilização do quartil $75 \%$ ou da média ponderada de todos os perfis, ou somente dos perfis a montante.

Os valores de referência local (VRL), a partir dos teores totais e disponíveis, foram calculados com os valores médios ponderados, considerando as seguintes opções de cálculo; (i) a média de todos os perfis, denominada média total, (ii) a média somente com os 
perfis 9,10 e 11, denominada média montante 1 , (iii) a média apenas com os perfis 9 e 10, denominada média montante 2 , e (iv) o quartil $75 \%$ de todos os perfis, considerando cada camada em particular $(0-$ $20,20-150$, e $0-150 \mathrm{~cm})$.

Os valores de referência obtidos pelo método holandês foram calculados a partir dos teores de argila e matéria orgânica das amostras, de acordo com a expressão:

$$
\mathrm{Zn}=[50+1,5(2 \mathrm{~L}+\mathrm{H})]
$$

em que $\mathrm{L}=\%$ de argila e $\mathrm{H}=\%$ de matéria orgânica.

Foram então calculadas as médias ponderadas para as profundidades de 0 - 150, 0 - 20 e $20-150 \mathrm{~cm}$. Essas médias foram consideradas valores de referência local (VRL) pelo método holandês.

Após a obtenção e escolha do valor de referência local ("background") para Zn, foram calculados o fator de contaminação (FC) e o índice de geoacumulação (IGeo). O FC é a razão entre a concentração do metal no solo e seu valor de referência (Laybauer, 1995), enquanto o IGeo é definido como medida quantitativa da poluição por metais pesados (Solomons \& Förstner, 1984). As fórmulas para o cálculo do FC e IGeo são:

$$
\mathrm{FC}=\mathrm{Cm} / \mathrm{Cn}
$$

$$
\text { Igeo }=\log _{2}[\mathrm{Cm} /(1,5 \times \mathrm{Cn})]
$$

em que $\mathrm{Cm}=$ concentração de determinado elemento no local de interesse;

$\mathrm{Cn}=$ concentração natural, "background" geoquímico ou valor de referência do elemento na área em estudo; 1,5 = fator usado para minimizar possíveis variações de "background" causadas por diferenças litológicas.

\section{RESULTADOS E DISCUSSÃO}

Os resultados apresentados a seguir referem-se às médias ponderadas dos teores observados nas camadas coletadas até os $20 \mathrm{~cm}$ superficiais e de $20-150 \mathrm{~cm}$, nos perfis dos solos.

\section{Teores totais e disponíveis de $\mathrm{Zn}$ nos solos}

Os solos sobre áreas mineralizadas (intacta ou minerada) apresentaram as maiores concentrações de Zn (Quadro 1) tanto na camada de 0 - $20 \mathrm{~cm}$ como na de 20 - $150 \mathrm{~cm}$ (perfis 1, 2, 3, 4 e 5), para todos os extratores, com destaque para o perfil 5 na camada de $0-20 \mathrm{~cm}$, e para os perfís 2 e 5 , na camada de $20-150 \mathrm{~cm}$.

\begin{tabular}{|c|c|c|c|c|c|c|}
\hline \multirow{4}{*}{ Perfil } & \multicolumn{4}{|c|}{ Teores disponiveis } & \multirow{2}{*}{\multicolumn{2}{|c|}{$\begin{array}{l}\text { Teores totais } \\
\mathrm{HF}-\mathrm{HNO}_{3}-\mathrm{HCl}\end{array}$}} \\
\hline & \multicolumn{2}{|c|}{ Mehlich-3 } & \multicolumn{2}{|c|}{ DTPA } & & \\
\hline & \multicolumn{6}{|c|}{ Camadas (cm) } \\
\hline & $0-20$ & 20-150 & $0-20$ & $20-150$ & $0-20$ & 20-150 \\
\hline \multicolumn{7}{|c|}{$\mathbf{m g ~ k g} \mathbf{~}^{-1}$} \\
\hline \multicolumn{7}{|c|}{ Área mineralizada não explorada } \\
\hline 1 & $2.617,12$ & 121,14 & 249,77 & 40,02 & $12.844,89$ & $6.288,83$ \\
\hline 2 & $3.721,98$ & $17.998,63$ & 434,51 & 518,07 & $15.286,18$ & $100.186,49$ \\
\hline 3 & $8.031,81$ & $7.400,30$ & 412,77 & 367,15 & $33.925,50$ & $30.819,35$ \\
\hline \multicolumn{7}{|c|}{ Área mineralizada em exploração } \\
\hline 4 & $11.187,15$ & $9.357,04$ & 536,48 & 464,70 & $52.935,82$ & $27.861,00$ \\
\hline 5 & $25.219,54$ & $21.082,23$ & 687,92 & 615,28 & $129.768,43$ & $93.956,83$ \\
\hline \multicolumn{7}{|c|}{ Área a jusante do empreendimento } \\
\hline 6 & 218,56 & 35,52 & 114,19 & 1,64 & $1.559,19$ & 898,77 \\
\hline 7 & 228,00 & 52,58 & 95,39 & 8,95 & $1.017,69$ & 430,37 \\
\hline 8 & 957,48 & 36,95 & 176,98 & 2,82 & $3.162,81$ & 431,70 \\
\hline \multicolumn{7}{|c|}{ Área a montante do empreendimento } \\
\hline 9 & 892,47 & 27,42 & 160,69 & 0,76 & $1.690,35$ & 513,89 \\
\hline 10 & 226,94 & 22,96 & 162,26 & 0,94 & 832,78 & 457,72 \\
\hline 11 & $4.750,82$ & 332,06 & 552,45 & 60,85 & $10.586,35$ & $3.084,58$ \\
\hline
\end{tabular}

Quadro 1. Médias ponderadas das concentrações de Zn, obtidas para as formas disponíveis (Mehlich-3 e DTPA) e total 
Para os demais perfis, em áreas a montante e a jusante da mina, os teores foram mais baixos, com exceção do perfil 11 (a montante) cujos teores das formas disponíveis na camada de $0-20 \mathrm{~cm}$ superam aqueles dos perfis sobre a área mineralizada não explorada (1 e 2). A localização desse perfil próximo e em altitude imediatamente inferior aos perfis que se encontram sobre a área em exploração (4 e 5), a superação dos valores somente na camada de 0 - 20 $\mathrm{cm}$ e apenas nas formas disponíveis levam a supor que esse perfil foi contaminado a partir das áreas vizinhas, possivelmente pelo escoamento superficial das águas de chuva ou por remobilização biogeoquímica.

Apesar dos altos teores de Zn no perfil 11, não se pode presumir contaminação desse solo por ação antrópica. Na descrição do perfil 11, verificou-se a presença de um horizonte A enterrado na profundidade de $18-23 \mathrm{~cm}$. A distribuição dos teores de $\mathrm{C}$ para esse perfil revela claramente isso. Verificase que o horizonte enterrado apresenta teor de $\mathrm{C}$ semelhante à camada superficial (Figura 2). Também fica claro que o aumento brusco nos teores totais de $\mathrm{Zn}$ na camada de $10-20 \mathrm{~cm}$ de profundidade não se deve apenas às formas residuais. Esse aumento pode ser creditado principalmente às formas ligadas a óxidos amorfos, carbonatos e matéria orgânica, que contribuem com cerca de 80 \% do total no horizonte A enterrado. Esses valores refletem-se em aumentos nos teores "biodisponíveis", extraídos por DTPA e Mehlich-3 (Figura 2). Além disso, a análise da camada de 18 - $23 \mathrm{~cm}$ em separado revelou teores de $\mathrm{Zn}$, de modo geral, superiores aos valores respectivos na camada superficial $(0-2 \mathrm{~cm})$. Isso significa contaminação do horizonte A enterrado, que era a superfície do solo em época pretérita, pretensamente anterior a qualquer atividade antrópica na região. Tal horizonte, contribuindo para aumento considerável das formas disponíveis em profundidade, cria condições para que as raízes da vegetação atual possam extrair e translocar Zn do horizonte enterrado para o atual horizonte A, caracterizando a ciclagem biogeoquímica.

Sabe-se que as formas mais lábeis (ou disponíveis) tendem a converter-se, com o tempo, em formas mais estáveis, diminuindo os teores disponíveis em relação ao total. Para Marques et al. (2002), a fração biodisponível de determinado metal pesado provavelmente será maior quando artificialmente adicionado ao solo do que quando ocorre naturalmente, de modo geral, na forma de minerais pouco solúveis. Portanto, a maior participação das formas lábeis, em relação ao total, na camada superficial do solo, pode ser considerada indício de contaminação recente, mais compatível com a ação antrópica. Deve-se, no entanto, observar todo o cenário onde a paisagem e a distribuição das formas disponíveis e totais nos perfis dos solos são aspectos importantes para discernir contaminação antrópica de anomalia natural. Assim, os teores totais e disponíveis de Zn e suas relações com a altitude e localização dos perfis são informações que se complementam no estudo de caracterização e diagnóstico da poluição. Nesse sentido, uma das contribuições do presente trabalho é sugerir que se considerem formas disponíveis como contaminação ativa e o teor total como a contaminação potencial em estudos detalhados de áreas suspeitas de contaminação.

\section{Fator de acumulação}

O fator de acumulação (FA) permite avaliação preliminar da contaminação por deposição ou acúmulo de material na superfície do solo, independentemente se por ação natural ou antrópica. Valores menores que um para o fator de acumulação $(\mathrm{FA}<1,0)$, em perfis que apresentarem concentrações maiores que o valor de referência local, confirmam que a área é naturalmente anômala. Nesse caso, não cabe suspeitar de contaminação antrópica.

De todos os solos estudados, o perfil 2 (área mineralizada não explorada) é o único a apresentar fator menor que um (Quadro 2), tanto para teores totais quanto para formas disponíveis, Mehlich-3 e DTPA $(0,15 ; 0,21$ e 0,84 , respectivamente), confirmando que a área é naturalmente anômala. Tal afirmação está de acordo com Marques et al. (2004), os quais verificaram que, com a exceção do Mn, não havia diferença entre os teores de elementostraço na superfície e em horizontes subsuperficiais de solos preservados da ação antrópica no Cerrado brasileiro. De fato, a localização do perfil 2 na paisagem mostra que não deve sofrer influência recente de outra área, mas pode influenciar as demais áreas pelo arraste de materiais sólidos e águas na época das chuvas.

Os perfis das áreas mineralizadas não explorados e em exploração (2, 3, 4 e 5) apresentaram baixo fator de acumulação (FA), em relação às áreas a montante e a jusante (Perfis 6, 7, 8, 9, 10 e 11). O perfil 1 apresentou FA's superiores aos demais perfís da área mineralizada, o que se deve à sua localização, próximo e abaixo dos perfis 2 e 3 .

Para as áreas a montante e jusante, os FA's calculados para os extratores Mehlich-3 e DTPA aumentam consideravelmente, indicando acúmulo superficial de Zn na fração disponível nessas áreas. Nesse sentido, o DTPA destaca-se como extrator mais sensível para distinguir as áreas naturalmente anômalas das contaminadas. Verifica-se, inclusive, que os perfis 1, na área mineralizada intacta, e 11, que apresenta um horizonte A enterrado a montante da área minerada, foram os únicos com FA's consideravelmente menores para o DTPA em relação ao Mehlich-3. 

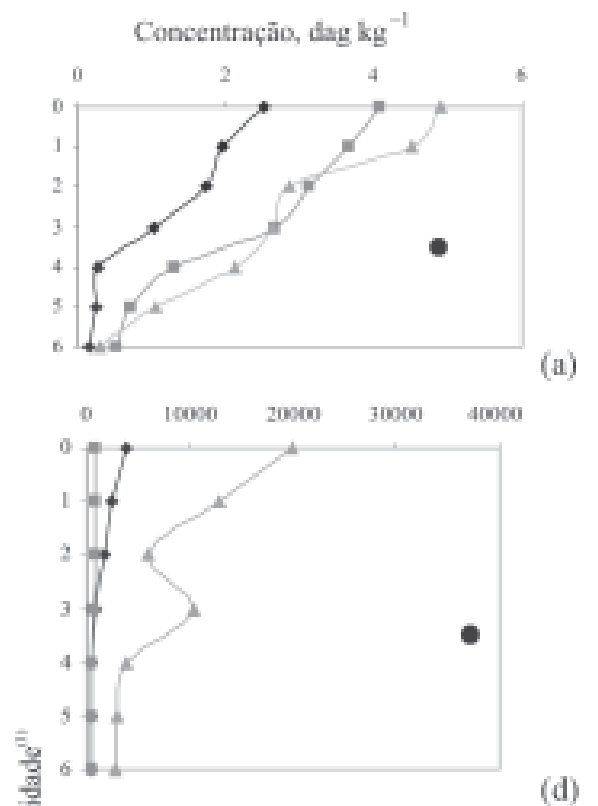

(d)

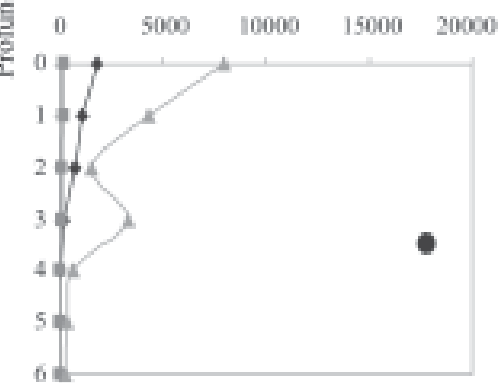

(g)

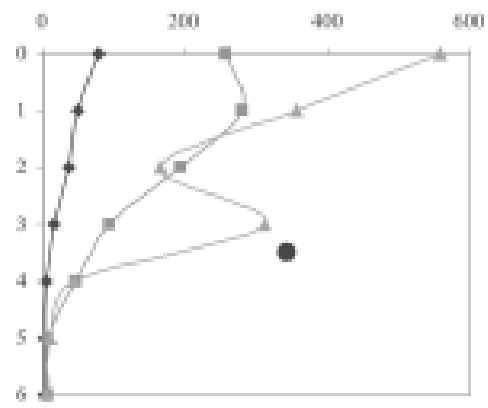

(j)
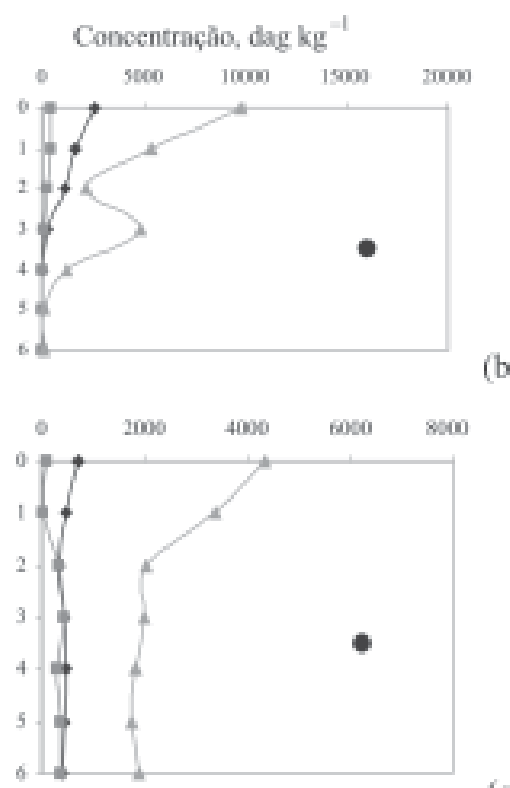

(b)

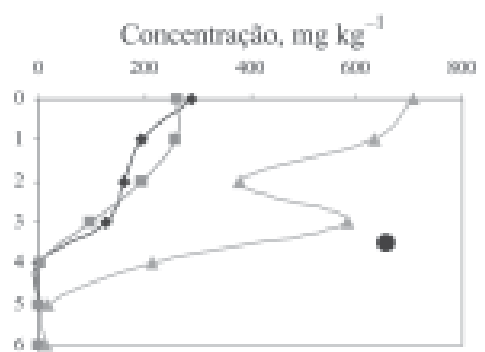

(c)

(e)

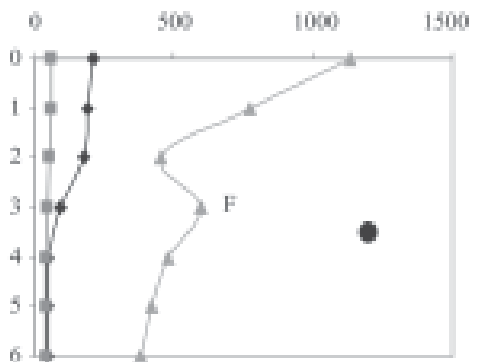

(f)

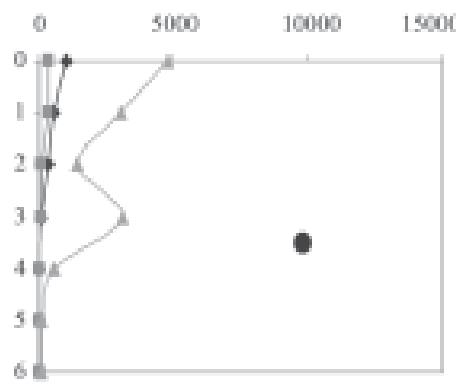

(h)

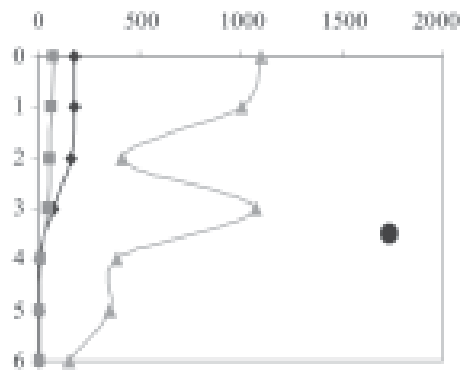

(i)

Figura 2. Teores de carbono orgânico e de Zn de acordo com a profundidade, nos perfis de solos da área em estudo. (a) - C orgânico; (b) - Zn extraído por Mehlich; (c) - Zn extraído por DTPA; (d) - Zn Total; (e) Fração Residual de Zn; (f) - Zn associado aos óxidos cristalinos; (g) - Zn associado aos óxidos amorfos; (h) - Zn associado a carbonatos; (i) - Zn associado à matéria orgânica; (j) - Zn trocável e l-Zn solúvel. (1) As profundidades $0,1,2,3,4,5$ e 6 correspondem a 0 - 2, 2 - 5, 5 - 10, 10 - 20, 20 - 50, 50 - 100 e 100 $150 \mathrm{~cm}$, respectivamente.

O diagnóstico de contaminação antrópica pressupõe que os altos teores no solo e o acúmulo de Zn na camada superficial não sejam naturais. Por outro lado, anomalias naturais geralmente caracterizam-se por: (a) baixas percentagens dos teores disponíveis em relação ao total e (b) possibilidade de acúmulo superficial por ciclagem biogeoquímica. Nesse caso, geralmente se verifica a presença de espécies acumuladoras na área, capazes de absorver o Zn de camadas profundas do solo com posterior deposição dos tecidos contaminados na superfície. A primeira condição (a) foi atestada em trabalho anterior (Borges Júnior et al., 2008) e pelos dados do presente trabalho. A condição (b) também pode ser considerada graças à presença de plantas indicadoras da presença de minério, denominadas "calamináceas", provavelmente hiperacumuladoras de Zn na área em estudo. 
Quadro 2. Fator de acumulação (camada superficial/ camada subsuperficial)

\begin{tabular}{|c|c|c|c|}
\hline \multirow[t]{2}{*}{ Perfil } & \multicolumn{3}{|c|}{ Fator de Acumulação } \\
\hline & Total & Mehlich-3 & DTPA \\
\hline \multicolumn{4}{|c|}{ Área mineralizada não explorada } \\
\hline 1 & 2,04 & 21,60 & 6,24 \\
\hline 2 & 0,15 & 0,21 & 0,84 \\
\hline 3 & 1,10 & 1,09 & 1,12 \\
\hline \multicolumn{4}{|c|}{ Área minerada em exploração } \\
\hline 4 & 1,90 & 1,20 & 1,15 \\
\hline 5 & 1,38 & 1,20 & 1,12 \\
\hline \multicolumn{4}{|c|}{ Área a jusante do empreendimento } \\
\hline 6 & 1,73 & 6,15 & 69,63 \\
\hline 7 & 2,36 & 4,34 & 10,66 \\
\hline 8 & 7,33 & 25,91 & 62,76 \\
\hline \multicolumn{4}{|c|}{ Área a montante do empreendimento } \\
\hline 9 & 3,29 & 32,55 & 211,43 \\
\hline 10 & 1,82 & 9,88 & 172,62 \\
\hline 11 & 3,43 & 14,31 & 9,08 \\
\hline
\end{tabular}

\section{Obtenção de valores de referência locais}

Os teores de Zn são, em geral, bastante elevados, principalmente na área mineralizada, indicando uma anomalia natural. Portanto, nessas condições, um valor de referência obtido a partir de dados locais não pode ser considerado referência de qualidade, mas sua determinação pode contribuir para avaliação da extensão e das causas da contaminação, bem como do risco ecotoxicológico. Assim, foram examinadas várias alternativas para a obtenção de um "valor de referência" ou "background" local.

Os valores de referência local obtidos são bastante diferentes, dependendo do extrator utilizado e dos perfis considerados (Quadro 3). Outro fator que determina variações nos valores obtidos é a profundidade, principalmente para as médias de perfis a montante. Portanto, há que se estabelecer uma profundidade que possa refletir o valor de referência local. Das quatro formas avaliadas (total, Mehlich-3, DTPA e fórmula da Holanda), tais valores de referência local obtidos pelo ataque total foram os maiores (Quadro 3), já que os valores obtidos pelo DTPA e Mehlich-3 consideram apenas o conteúdo das formas pretensamente disponíveis do elemento no solo. No caso do método baseado na fórmula da Holanda, os valores de referência calculados foram menores, já que não se referem a áreas anômalas e, obviamente, não refletem as condições locais.

Quadro 3. Valores de referência de Zn, para o município de Vazante-MG, obtidos por diferentes métodos e profundidades

\begin{tabular}{|c|c|c|c|c|}
\hline Profundidade & Média Total & Média Montante 1 & Média Montante 2 & Quartil $75 \%$ \\
\hline $\mathrm{cm}$ & \multicolumn{4}{|c|}{ Total (mg kg-1) } \\
\hline $0-20$ & $23.964,55$ & $4.369,83$ & $1.261,57$ & $24.887,89$ \\
\hline $20-150$ & $24.112,61$ & $1.352,06$ & 485,81 & $27.401,75$ \\
\hline \multirow[t]{2}{*}{$0-150$} & $24.092,87$ & $1.754,43$ & 589,24 & $27.066,57$ \\
\hline & \multicolumn{4}{|c|}{ Mehlich-3 (mg kg-1) } \\
\hline $0-20$ & $5.100,43$ & $1.956,74$ & 559,71 & $7.021,98$ \\
\hline $20-150$ & $5.062,64$ & 127,48 & 25,19 & $7.172,16$ \\
\hline \multirow[t]{2}{*}{$0-150$} & $5.067,67$ & 371,38 & 96,46 & $7.152,13$ \\
\hline & \multicolumn{4}{|c|}{ DTPA (mg kg-1) } \\
\hline $0-20$ & 325,77 & 291,80 & 161,48 & 487,61 \\
\hline $20-150$ & 178,75 & 20,85 & 1,48 & 360,14 \\
\hline \multirow[t]{2}{*}{$0-150$} & 198,35 & 56,98 & 22,81 & 377,14 \\
\hline & \multicolumn{4}{|c|}{ Fórmula da Holanda (mg kg-1) } \\
\hline $0-20$ & 162,03 & 214,33 & 237,57 & 224,03 \\
\hline $20-150$ & 152,85 & 193,02 & 220,44 & 172,97 \\
\hline $0-150$ & 163,19 & 217,61 & 240,20 & 231,81 \\
\hline
\end{tabular}


Os teores disponíveis poderiam ser preferidos em relação aos teores totais, para a determinação dos valores de referência local. No entanto, considerando que, de modo geral, os métodos utilizados consideram a extração com água régia, acredita-se que o valor de referência obtido pelo ataque total é, ainda, a melhor opção para comparações com outras tabelas de valores de referência.

Tanto o quartil $75 \%$ quanto a média dos teores de $\mathrm{Zn}$ de todos os perfis apresentaram valores mais elevados, seguidos pela média montante $1 \mathrm{e}$ 2 (Quadro 3). Isso obviamente decorre de teores mais altos de $\mathrm{Zn}$ para os perfis sobre as áreas mineralizadas, em relação às áreas a montante. Por outro lado, a exclusão do perfil 11, com indícios de contaminação, para cálculo dos teores médios a montante, diminuiu, consideravelmente, os valores de referência. Desse modo, optou-se pela média montante 2 , para representar o valor de referência local, visto que as demais, teoricamente, estão sob influencia direta da área mineralizada.

Na discussão do fator de acumulação, verificouse que, em áreas anômalas, pode haver maior teor na camada superficial do solo, causada por ciclagem biogeoquímica. Assim sendo, e considerando que, de modo geral, os estudos de avaliação da contaminação referem-se à profundidade de $0-20 \mathrm{~cm}$, considerase que os valores de referência obtidos para esta profundidade sejam mais adequados.

Optando pelo método de extração total, os perfis 9 e 10 (montante) e a camada de $0-20 \mathrm{~cm}$ são as condições que melhor representam a área. Com vistas em determinar os valores de referência comparáveis com a literatura, obteve-se: $1.262 \mathrm{mg}$ $\mathrm{kg}^{-1}$ como valor de referência local (VRL) para Zn. Este valor é muito superior ao valor de referência de qualidade (VRL $\cong 21 \times \mathrm{VRQ}$ ) e ao valor de alerta adotados no estado de São Paulo (Quadro 4). Por outro lado, é inferior aos valores de intervenção (VI) para áreas industriais adotados no Estado de São Paulo, EUA e Canadá.

Ao comparar valores de referência locais com valores orientadores obtidos para outras regiões, devem-se considerar as limitações impostas pelas diferenças regionais, bem como por diferenças metodológicas. É importante esclarecer que o método de extração (água régia) adotado no Estado de São Paulo, apesar de extrair a maior parte dos metais do solo, não solubiliza completamente as formas ligadas a silicatos. Por outro lado, o método utilizado no presente trabalho (ataque triácido HF$\mathrm{HNO}_{3}-\mathrm{HCl}$ em forno microondas) promove, também, dissolução dos silicatos. Em que pesem as limitações de comparar VRL de uma região anômala com VR's de outras localidades, considerase que tais comparações são úteis na interpretação dos valores da área em estudo. No caso presente, o VRL ultrapassa os valores de intervenção (VI) para o cenário agrícola e residencial, adotados em São Paulo e no Canadá. Portanto, considerando que o VI depende mais de variáveis relacionadas com exposição humana do que das características do meio físico, pode-se admitir risco à saúde humana para uso agrícola e residencial da área em estudo.

Quadro 4. Comparação do valor de referência de Zn para a região de Vazante com valores orientadores para o Estado de São Paulo e Internacionais

\begin{tabular}{|c|c|c|c|c|c|}
\hline \multicolumn{2}{|c|}{ Índice } & Vazante-MG & $\begin{array}{l}\text { Estado de } \\
\text { São Paulo }\end{array}$ & $\begin{array}{c}\text { Canadá } \\
\text { (Federal) }\end{array}$ & $\begin{array}{c}\text { Estados Unidos } \\
\text { da América }\end{array}$ \\
\hline \multicolumn{2}{|c|}{ Valor de Referência Local } & 1.262 & - & - & - \\
\hline \multicolumn{2}{|c|}{ Valor de Referência de Qualidade } & - & 60 & - & - \\
\hline \multicolumn{2}{|c|}{ Valor de Alerta } & - & 300 & - & - \\
\hline \multirow{3}{*}{$\begin{array}{l}\text { Valor de } \\
\text { Intervenção }\end{array}$} & Agrícola & - & 500 & - & - \\
\hline & Residencial & - & 1.000 & - & - \\
\hline & Industrial & - & 1.500 & - & - \\
\hline \multicolumn{2}{|l|}{ Agrícola } & - & - & 600 & - \\
\hline \multicolumn{2}{|l|}{ Residencial } & - & - & 500 & 23.000 \\
\hline \multicolumn{2}{|l|}{ Intervenção } & - & - & 1.500 & 310.000 \\
\hline
\end{tabular}


Fator de contaminação e índice de geoacumulação (IGeo)

Os perfis sobre a área mineralizada apresentaram os FC's mais elevados, com destaque para o perfil 5 com $\mathrm{FC} \cong 103$, calculado a partir dos teores totais (Quadro 5). No caso das áreas a jusante, o valor foi alto para o perfil $8(\mathrm{FC}=2,51)$ e o perfil 7 apresentou FC menor que 1, considerando os teores totais. O perfil 8 encontra-se à beira de uma estrada, a jusante da mineração, de modo que existe a possibilidade de contaminação por atividade antrópica. Por outro lado, o perfil 7 é um solo aluvial às margens do Rio Santa Catarina, formado a partir de depósitos recentes, a jusante da mina, que não apresenta sinais de contaminação.

Os resultados também confirmam suspeita de contaminação do perfil 11 ( $\mathrm{FC}=8,39$ para teores totais). Sua localização a montante da mineração não revela contaminação por atividade antrópica. Portanto, esse perfil foi excluído da média para obtenção do VRL, em razão da suspeita de contaminação, conforme discutido anteriormente.

Os fatores de contaminação (FC's) calculados a partir dos teores extraíveis por DPTA foram menores em relação aos calculados com teores totais. Por outro lado, os FC's calculados para Mehlich-3 foram maiores do que os totais. No entanto, os FC's para teores disponíveis não modificam substancialmente as observações feitas com base em teores totais. Apenas o DTPA destaca a suspeita de contaminação do perfil 11 , pois altera consideravelmente sua situação em relação aos demais, com FC na mesma ordem de magnitude dos perfis das áreas mineradas. Tais resultados, a exemplo do que foi discutido anteriormente, novamente mostram maior sensibilidade do extrator DTPA em relação aos demais para distinguir anomalias geoquímicas de áreas contaminadas.

De acordo com o IGeo (Quadros 5 e 6), os solos a jusante da área minerada foram considerados não poluídos a moderadamente poluídos. Por outro lado, os solos das áreas mineralizadas mostraram-se moderadamente a exageradamente poluídos. Dentre esses, destacam-se os perfis 3 e 4 na classe 5 (fortemente a exageradamente poluído) e o perfil 5 na classe 6 (exageradamente poluído). Tais resultados, novamente, confirmam que os solos a jusante do empreendimento não se encontram poluídos ou contaminados por ação antrópica, com exceção do perfil 8 , o qual pode ser considerado moderadamente poluído.

Quadro 5. Fator de contaminação (FC) e índice de geoacumulação (IGeo) de Zn em área minerada na região de Vazante-MG

\begin{tabular}{lccccccccc}
\hline Característica & \multicolumn{10}{c}{ Perfil } \\
\hline & $\mathbf{1}$ & $\mathbf{2}$ & $\mathbf{3}$ & $\mathbf{4}$ & $\mathbf{5}$ & $\mathbf{6}$ & $\mathbf{7}$ & $\mathbf{8}$ & $\mathbf{1 1}$ \\
FC (Total) & 10,18 & 12,12 & 26,89 & 41,96 & 102,86 & 1,24 & 0,81 & 2,51 & 8,39 \\
FC (Mehlich) & 16,21 & 23,05 & 49,74 & 69,28 & 156,18 & 1,35 & 1,41 & 5,93 & 29,42 \\
FC (DTPA) & 1,55 & 2,69 & 2,56 & 3,32 & 4,26 & 0,71 & 0,59 & 1,10 & 3,42 \\
IGeo & 2,8 & 3,0 & 4,2 & 4,8 & 6,1 & $-0,3$ & $-0,9$ & 0,7 & 2,5 \\
\hline
\end{tabular}

Quadro 6. Classificação do Índice de Geoacumulação de acordo com a acumulação de Zn no sedimento, segundo Förstner \& Müller (1981)

\begin{tabular}{clcc}
\hline Classes IGeo & \multicolumn{1}{c}{ Intensidade da Poluição } & Acúmulo no Sedimento & Perfis \\
\hline 0 & Não poluído & $<0$ & 6 e 7 \\
1 & Não poluído a moderadamente poluído & $>0-1$ & 8 \\
2 & Moderadamente poluído & $>1-2$ & - \\
3 & Moderadamente a fortemente poluído & $>2-3$ & 1,2 e 11 \\
4 & Fortemente poluído & $>3-4$ & - \\
5 & Fortemente a exageradamente poluído & $>4-5$ & 3 e 4 \\
6 & Exageradamente poluído & $>5$ & 5 \\
\hline
\end{tabular}




\section{CONCLUSÕES}

1. Os perfis das áreas mineralizadas, de modo geral, não revelaram acúmulo de Zn na superfície. Para áreas a montante e a jusante, o fator de acumulação aumentou muito, principalmente para extratores das formas disponíveis.

2. O valor de referência local (VRL) obtido para Zn, muito superior ao valor de referência de qualidade adotado para o Estado de São Paulo, sugere monitoramento e restrição ao uso agrícola e residencial dos solos na área. Não obstante, o VRL obtido é menor que os valores de intervenção (VI) para áreas industriais, adotados no Estado de São Paulo, EUA e Canadá.

3. Os solos da área mineralizada apresentaram os maiores fatores de contaminação para Zn, com destaque para o Cambissolo/Neossolo Húmico da área minerada (perfil 5). Nos solos das áreas a jusante, os valores mais altos foram obtidos para o Latossolo Vermelho-Escuro (perfil 8) e o mais baixo para o Neossolo Flúvico (perfil 7).

4. Os solos a jusante podem ser considerados não poluídos a moderadamente poluídos, segundo o índice de geocumulação (IGeo) calculado com base no VRL. Nesse sentido, destaca-se o perfil 8 como sendo o único no qual se pode presumir contaminação antrópica.

4. O DTPA destacou-se como extrator mais sensível para distinguir áreas anômalas de áreas contaminadas.

\section{LITERATURA CITADA}

BORGES JÚNIOR, M.; MELLO, J.W.V.; SCHAEFER, C.E.G.R.; DUSSIN, T.M. \& AMIM JÚNIOR, J. Distribuição e formas de ocorrência zinco em solos no município de Vazante-MG.. R. Bras. Ci Solo 32:2183-2194, 2008.

COMPANHIA DE TECNOLOGIA DE SANEAMENTO AMBIENTAL - CETESB. Relatório de estabelecimento de valores orientadores para solos e águas subterrâneas no Estado de São Paulo. São Paulo, 2001. 247p.

COMPANHIA DE TECNOLOGIA DE SANEAMENTO AMBIENTAL - CETESB. Manual de gerenciamento de áreas contaminadas. Projeto CETESB-GTZ. Cooperação Técnica Brasil-Alemanha. 2.ed. São Paulo, 1999. 389p.
EMPRESA BRASILEIRA DE PESQUISA AGROPECUÁRIA . EMBRAPA. Manual de análises químicas de solos, plantas e fertilizantes. Brasília, Embrapa Comunicação para Transferência de Tecnologia, 1999. 370p.

LAYBAUER, L. Análise de transferência de metais pesados em águas e sedimentos fluviais na região das Minas de Camaquã. Porto Alegre, Universidade Federal do Rio Grande do Sul, 1995. 146p. (Tese de Mestrado)

LINDSAY, W.L. \& NORVEL, W.A. Development of a DTPA soil test for zinc, iron, manganese and copper. Soil Sci. Am. J., 49:597-603, 1978.

MARQUES, J.J.G.S.M.; CURI, N. \& SCHULZE, D.G. Trace elements in Cerrado soils. In: ALVAREZ V., V.H.; SCHAEFER, C.E.G.R.; BARROS, N.F.; MELLO, W.V. \& COSTA, L.M., eds. Tópicos em ciência do solo. Viçosa, MG, Sociedade Brasileira de Ciência do Solo, 2002. v.2. p.103-142.

MARQUES, J.J.; SCHULZE, D.G.; CURI, N. \& MERTZMAN, S.A. Trace elements geochemistry in Brazilian Cerrado soils. Geoderma, 121:31-34, 2004.

MEHLICH, A. Mehlich 3 soil test extractant: A modification of mehlich 2 extractant. Comm. Soil Sci. Plant Anal., 15:14091416, 1984.

RAURET, G. Extraction procedures for the determination of heavy metals in contaminated soil and sediment. Talanta, 46:449-455, 1998

RIBEIRO FILHO, M.R.; CURI, N.; SIQUEIRA, J.O. \& MOTTA, P.E.F. Metais pesados em solos de área de rejeitos de indústria de processamento de zinco. R. Bras. Ci. Solo, 23:453-464, 1999.

RIGOBELLO, A.E.; BRANQUINHO, J.A.; DANTAS, M.G.S.; OLIVEIRA, T.F. \& NIEVES FILHO, W. Mina de zinco de Vazante, Minas Gerais. In: BRASIL. Ministério das Minas e Energia, DNPM/CVRD. Principais dépósitos minerais do Brasil. v.2. 1988. 670p.

SOLOMONS, W. \& FÖRSTNER, U. Metals in the hydrocycle. Berlin, Springer Verlag, 1984.

UNITED STATES ENVIRONMENTAL PROTECTION AGENCY - USEPA. Soil screening guidance: User's guide - EPA 450/R-96/018. Washington, Office of Solid Waste and Emergency Response, 1996.

VISSER, W.J.F. Contaminated land policies in some industrialized countries. 2.ed. The Hague, Technical Soil Protection Committee, 1994. 149p.

VROM. Intervention values and target values: Soil quality standards. The Hague, 1994. 19p. (DBO/07494013) 
